\title{
Société Française de Physique Le point sur les actions menées en 2013
}

En ce retour de vacances, il me semble judicieux de faire le point sur les diverses actions menées en 2013, sur leur succès ou leur degré d'avancement. Voici les plus importantes.

\section{- Le Congrès général de Marseille}

Nous ne disposons pas encore des chiffres définitifs, mais on peut d'ores et déjà affirmer que celui-ci a été un succès. Je tiens à remercier l'organisateur Dave Lollmann, ainsi que toute l'équipe qui l'a aidé. Le nombre de participants a été de l'ordre de 600. Le congrès s'est déroulé dans un cadre tout à fait agréable. Les conférences plénières ont été très appréciées. Un compte-rendu quantitatif vous sera présenté dans le prochain numéro.

- Site web

Une équipe menée par Kees van der Beek a réalisé avec la société Jouve un travail de fond concernant le cahier des charges. Celui-ci est maintenant terminé, mais le devis proposé pour la réalisation n'est pas acceptable. Nous avons donc mis plusieurs sociétés en concurrence et espérons pouvoir prendre une décision d'ici un mois. Notre objectif est ambitieux, mais l'avenir de la SFP en dépend.

- L'organisation du siège

L'année qui vient verra de profondes évolutions. Tout d'abord le CEA, que nous remercions pour cette décision très positive, vient de mettre Jean-Jacques Bénattar à la disposition de la SFP, et ceci à plein temps. Dans le même temps, Gérard Bonneaud, qui avait accepté la lourde tâche de secrétaire général, a décidé de démissionner. Nous venons donc d'obtenir l'accord du Conseil d'administration du 20 septembre pour que J.J. Bénattar devienne secrétaire général, avec l'avantage qu'il sera présent de manière permanente au siège. Par ailleurs, Véronique Lemaître va nous quitter en juillet 2014 et nous devrons pourvoir à son remplacement. Enfin, il nous faudra un permanent, au moins à mi-temps, pour assurer un suivi et une alimentation corrects du site Web. Tout ceci est à l'étude et devrait aboutir à une organisation plus vivante du siège de la SFP.

- EDP Sciences

Nous allons organiser quelques minibureaux pour étudier comment la SFP peut accompagner les projets d'évolution d'EDP Sciences, en particulier en ce qui concerne le développement de l'Open Access Gold.

- Les relations avec les autres sociétés savantes

Sous l'impulsion de notre vice-président Alain Fontaine, nous entamons un rapprochement avec la Société Chimique de France et la Société Française d'Optique, de manière à mieux coordonner certaines actions et à travailler sur des projets communs. Par ailleurs, nous avons publié en commun avec l'UdPPC un numéro spécial de Reflets de la physique et du BUP sur le rayonnement synchrotron. Ce numéro donne un aperçu extrêmement complet et abordable des possibilités offertes par ces grands instruments.

- L'évolution des EPAM (Écoles de Physique Avancée au Maghreb)

Alain Fontaine a esquissé un projet autour d'un programme d'écoles scientifiques annuelles, ciblées sur l'usage du rayonnement synchrotron et sur les disciplines expérimentales nécessaires à la fabrication et l'exploitation de cette source de lumière unique. Ceci se fera dans le cadre d'une approche partenariale du GID (Groupement Interacadémique pour le Développement), de la Société Française de Physique et de la Société Chimique de France.

- La politique éditoriale SFP-CNRS

La nouvelle politique éditoriale, inaugurée en janvier 2013 (lors du numéro 32), se poursuivra cette année et début 2014, avec inclusion dans Reflets de quelques articles initialement prévus pour Images de la Physique. Simultanément, se mettra en place un comité de rédaction commun, qui présidera aux destinées de la revue Reflets de la physique élargie. Signalons d'ailleurs que le présent numéro contient une lettre ouverte à ce sujet (p. 47).

Comme vous pouvez le constater, un certain nombre d'évolutions importantes sont en cours. Nous n'avons pas la place d'aborder ici tous les sujets, mais nous vous informerons régulièrement des résultats obtenus. 